\title{
Multiple Intracardiac Masses as the Primary Presentation of a Systemic Inflammatory Disease: A Big Challenge (Case Report)
}

\author{
Zahra Khalaji ${ }^{1}$, Bahar Galeshi ${ }^{1}$, simin almasi ${ }^{1}$, Mozhgan Parsaee ${ }^{1}$, and Hamidreza \\ Pouraliakbar ${ }^{1}$ \\ ${ }^{1}$ Iran University of Medical Sciences
}

January 20, 2021

\begin{abstract}
Antiphospholipid syndrome (APS) classically presents with venous or arterial thrombosis and pregnancy morbidity. Although clinical manifestations with fever of unknown origin and intracardiac masses are unusual, in a patient with prolonged fever and multiple intracardiac thrombi, systemic inflammatory diseases such as APS should be considered.
\end{abstract}

\section{INTRODUCTION}

Intracardiac masses are usually detected incidentally during echocardiography or in the setting of a systematic approach to a patient's symptoms. The most common entities are thrombi, vegetation, and to a lesser extent, cardiac tumors ${ }^{1}$; nonetheless, on the rare occasion of the presence of multiple intracardiac masses, all of them should be considered in the differential diagnosis. ${ }^{2}{ }^{3}$ Intracardiac thrombosis (ICT) is commonly detected in the presence of a predisposing factor; in some instances, however, it implies a potentially underlying serious condition, the most notable instance of which is the possibility of systemic inflammatory diseases in the presence of right heart thrombi. ${ }^{3,4}$ Antiphospholipid syndrome (APS) is a heterogeneous autoimmune disease that is characterized by vascular thrombosis, complicated pregnancy, and elevated antiphospholipid antibodies. Although valvular disease is the most common presentation of APS, ICT may be the main clinical manifestation of this systemic inflammatory disease. ${ }^{5}$ We herein describe a young man with a diagnosis of APS who presented with fever and multiple intracardiac masses. Besides highlighting this rare presentation, we underscore the diagnostic and therapeutic value of inflammatory thrombosis.

\section{CASE PRESENTATION}

A previously healthy 38-year-old man was referred to our emergency department with a diagnosis of a cardiac mass according to 2D echocardiography. The patient complained of intermittent fever, loss of appetite, and unintentional weight loss ([?] $8 \mathrm{~kg}$ ) within the preceding 3 months. His history was negative for similar prior episodes and symptoms related to the gastrointestinal tract or the pulmonary and urinary systems. Additionally, he had no complaints of headache and other neurologic symptoms, myalgia, arthralgia, arthritis, or even mucocutaneous manifestations. Still, he had a history of recurrent painful oral aphthosis without a history of genital ulcers. The physical examination revealed a low-grade fever $\left(38.5^{\circ} \mathrm{C}\right.$ oral $)$, a blood pressure of 120/80 $\mathrm{mm} \mathrm{Hg}$, and normal sinus rhythm. The cardiopulmonary examination was unremarkable. The lower limbs were symmetric in size and pulse without edema. The rheumatologic and neurologic examinations showed no positive findings.

Blood culture and complete workup were done for the evaluation of malignancy and hypercoagulable state. Despite the remote possibility of endocarditis, empirical antibiotics were administered. The complete blood count showed thrombocytopenia (platelet count $=104 / \mathrm{L}$ ) and anemia (hemoglobin $=11.3 \mathrm{~g} / \mathrm{dL}$ ) without 
leukocytosis. The activated partial thromboplastin time was within the normal limit. The results of comprehensive thrombophilia and rheumatologic workup revealed only a mild elevation in the homocysteine level with weakly positive anti- $\beta 2$ glycoprotein (anti- $\beta 2 \mathrm{GPI}$ ). The pathergy test to evaluate Behçet's disease was also negative.

Given the patient's hypercoagulopathy state in the current COVID-19 pandemic, a polymerase chain reaction test was performed, which was negative. The most important lab data are summarized in Table 1.

Parallel to the laboratory evaluation, an imaging study was conducted. Comprehensive 2D transthoracic echocardiography, followed by transesophageal echocardiography, visualized multiple intracardiac masses (Table 2): a large lobulated intracavity mass (maximum size $=6 \times 3 \mathrm{~cm}$ ) in the right ventricle (RV) with extension to RV apex, a large homogenous fixed right atrial (RA) mass $(4 \times 3 \mathrm{~cm})$ in the superior part of RA roof, and a small round left ventricular (LV) apical mass $(6 \times 9 \mathrm{~mm})$. In addition, a plethoric inferior vena cava (IVC) with a smoky pattern and a large lobulated thrombus ([?] $6 \mathrm{~cm}$ ) with extension to RA-IVC junction was noticeable (Fig. 1). In the first step, deep venous thrombosis and pulmonary embolism were ruled out. Multi-slice axial chest computed tomography (CT) was normal, except for the presence of masses in the chambers, which was compatible with the echocardiographic findings. Comprehensive abdominopelvic sonography revealed normal findings, with the exception of moderate ascites.

The initial clinical and paraclinical assessments ruled out infectious endocarditis and secondary tumors. Further, given the rarity of multiple myxomas and the patient's insignificant response to parenteral anticoagulation, the most probable diagnosis was inflammatory thrombosis, APS, or Behcet's disease. Notwithstanding that our findings could not fulfill the diagnostic criteria of any one of them, interestingly, ICT, especially right heart involvement, could be the first presentation of systemic inflammatory diseases, and in particular Behcet's disease.

For the establishment of the diagnosis, cardiac magnetic resonance imaging was performed using a $1.5 \mathrm{~T}$ Siemens Avanto machine (Fig. 2). Cine sequences were obtained using balanced steady-state free precession (bSSFP), perfusion sequences, and delayed enhancement (turbo field echo with inversion pulse and recovery, 10 minutes after the injection of $0.2 \mathrm{mmol} / \mathrm{kg}$ of the gadolinium contrast medium). Filling defects were detected in the apex of RV $(3 \times 2.1 \mathrm{~cm})$, LV $(0.6 \times 0.56 \mathrm{~cm})$, and RA $(4 \times 3 \mathrm{~cm})$. Large elongated filling defects were also depicted in the hepatic portion of IVC that extended toward RA. Additionally, RV apex was obliterated by the intracardiac mass. Based on different sequences and tissue characterizations, the findings of the cardiac magnetic resonance imaging were compatible with intracardiac thrombosis. Moreover, subendocardial late gadolinium enhancement was observed in the biventricular apical portions in the affected segments, suggestive of endomyocardial fibrosis.

With a diagnosis of APS and under the supervision of a rheumatologist, the patient received antiinflammatory treatment with corticosteroid pulse therapy at a dose of $500 \mathrm{mg}$ for 3 days, intravenous cyclophosphamide monthly, and ASA $80 \mathrm{mg}$ daily.

The patient's clinical status was improved, and his fever was resolved. Transthoracic echocardiography demonstrated a relative reduction in the size of the RA/RV mass, with the complete elimination of the $\mathrm{LV}$ apical thrombus. In addition, during the course of treatment, abdominopelvic CT scanning illustrated abdominal arterial/venous thrombosis with pelvic extension. The late occurrence of these common features of APS was a strong confirmation of the diagnosis of this syndrome. The extensive thrombosis necessitated high-dose medication. Follow-up echocardiography showed a significant reduction in the size of the RV/RA mass, with a remarkable improvement in LV/RV function. Additionally, the IVC mass was also eliminated.

Up to the time of writing this paper, the close monitoring of the patient revealed no new findings.

\section{DISCUSSION}

The cardiovascular system can be affected during systemic inflammatory and rheumatologic disorders. ${ }^{2,6}$ Valvular disease, conduction disturbances, myocardial and pericardial involvement, and also vasculitis can be detected. Similarly, intracardiac masses associated with a systemic inflammatory disease such as Wegener's 
granulomatosis, sarcoidosis, rheumatoid arthritis, and Behcet's disease are reported. ${ }^{2}{ }^{6}$ ICT may develop in the presence of a known systemic disorder or might be the isolated and first clinical presentation. ${ }^{2}$ APS syndrome or Hughes' syndrome is a rare prothrombotic autoimmune disease characterized by recurrent vascular thrombosis events (arterial, venous, or even microvascular circulation), fetal loss, and persistent (at least 12 weeks apart) elevated levels of antiphospholipid antibodies, including the lupus anticoagulant (LA), and /or moderate-to-high levels of the isotypes of immunoglobulin M (IgM) or immunoglobulin G (IgG) anticardiolipin (aCL) antibodies or the isotypes of IgG or IgM anti- $\beta 2 \mathrm{GPI}$, leading to a hypercoagulable state and related symptoms.(1-4) The diagnosis is confirmed in the presence of at least 1 clinical and 1 laboratory criteria. The annual incidence of APS was estimated at around 5 new cases per 100000 people. ${ }^{9}$ This syndrome may be developed as a primary condition or a secondary disorder related to an underlying disease, most commonly systemic lupus erythematosus. Mavrogeni et $\mathrm{al}^{5}$ described cardiac involvement as a common complication found in $30 \%$ of patients following APS. Also, this syndrome, itself, was considered a risk factor for cardiovascular disease. ${ }^{10}$ Cardiac complications are categorized into 2 main subgroups of valvular heart disease (more common $)^{5,11}$ and non-valvular involvement (less frequent), including ICT, myocardial disease, LV and RV dysfunction, and pulmonary hypertension. ${ }^{5}$ Cardiac thrombosis is an uncommon complication ${ }^{(4-7)}$ and is responsible for $1.9 \%$ of all cases of ICT. ${ }^{15}$ Albeit ICT is a life-threatening complication, appropriate treatment may decrease its morbidity and mortality rates. Ye Z-X et al ${ }^{16}$ described a 22 -year-old woman with an RA thrombus, deep vein thrombosis/pulmonary thromboembolism, and positive aCL antibodies. Voigtlaender et al $^{17}$ described a 67 -year-old woman with an RA thrombus, thrombocytopenia, and positive LA antibodies. Zahid et $\mathrm{l}^{18}$ reported a case of an RA thrombus and pulmonary thromboembolism, as the first presentations of APS, in a 26-year-old man confirmed by positive LA/aCL antibodies.

In 2011, Permanyer et al ${ }^{19}$ described a 43-year-old woman with a cardiac mass at the injunction of RA to IVC (dilated IVC), which was finally was diagnosed as APS syndrome due to strongly positive LA/aCL antibodies. In a literature review conducted by Waisayarat et $\mathrm{al}^{9}$ on 9 patients with ICT and APS, most of the patients were women aged between 30 and 40 years old. The heart chamber with the most involvement was RA, and LA and LV were less commonly involved. LA antibodies, followed by aCL antibodies, were positive in most cases, and anti- $\beta 2 \mathrm{GPI}$ was positive in only 2 cases.

In another literature review, Dhibar et $\mathrm{al}^{20}$ showed that fever of unknown origin was a rare presentation in primary APS. Their analysis on 28 cases presenting with ICT showed a relatively female predominance with more common right heart involvement, not least RA, and to a lesser extent, LA or LV involvement. Only 2 cases had a simultaneous involvement of LV/LA and right heart. Primary APS was more prevalent, and in most cases LA/aCL antibodies were positive. Positive anti- $\beta 2$ GPI was reported in only 3 patients, all of whom had an RA mass and positive LA and/or aCL antibodies. In contrast to these case reports, our patient, who was a man presenting with extensive ICT and simultaneous RA/RV/LV/and IVC masses, had anti- $\beta 2$ GPI as the only positive antibody in the laboratory assessment. Such discrepancies with prior cases prompted us to report this case as a relatively strange example. It is probable that in the follow-up of our patient, other groups of positive antibodies may be detected.

\section{CONCLUSIONS}

In the setting of prolonged fever, intracardiac masses, negative thrombophilia workup, inappropriate response to parenteral anticoagulation, and the absence of the underlying etiologies of thrombus formation, the most probable diagnosis in our patient was inflammatory ICT, as the main clinical manifestation of APS. Scintillatingly, even the initial rheumatologic workup was negative.

\section{Author contribution:}

Z.Khajali and S.Almasi: involved in patient management and design of the study

B.Galeshi: participated in acquiring patient data, performing the literature review and writing the draft of manuscript

M.Parsaee and HR.Pouraliakbar: interpretation and preparation of echocardiography and cardiac MRI data 
respectively

All authors read and approved the final manuscript.

Acknowledgement statement:

We thank to Dr Habib. Rohban help us in literature review.

Conflict of interes:

No conflict of interest.

\section{REFERENCES}

1. Mavrogeni SI, Sfikakis PP, Kitas GD, Kolovou G, Tektonidou MG, editors. Cardiac involvement in antiphospholipid syndrome: The diagnostic role of noninvasive cardiac imaging. Seminars in Arthritis and Rheumatism; 2016: Elsevier.

2. Zayed S, Tlili R, Azaiez F, Hentati R, Zouari F, Romdhane RB, et al. An Unusual Cause of Multiple Left Ventricle Thrombi in a Young Woman. Journal of Case Reports. 2020;10(3):189-91.

3. Polytarchou K, Varvarousis D, Manolis AS. Cardiovascular Disease in Antiphospholipid Syndrome. Current Vascular Pharmacology. 2020.

4. Waisayarat J, Plumworasawat S, Vilaiyuk S, Sirachainan N. Intracardiac thrombus in a patient with catastrophic antiphospholipid syndrome: an autopsy case report and review of the literature. Vascular health and risk management. 2019;15:253.

5. Cianciulli TF, Saccheri MC, Redruello HJ, Cosarinsky LA, Celano L, Trila CS, et al. Right atrial thrombus mimicking myxoma with pulmonary embolism in a patient with systemic lupus erythematosus and secondary antiphospholipid syndrome. Texas Heart Institute Journal. 2008;35(4):454.

6. Cianciulli TF, Saccheri MC, Lax JA, Neme RO, Sevillano JFA, Maiori ME, et al. Left ventricular thrombus mimicking primary cardiac tumor in a patient with primary antiphospholipid syndrome and recurrent systemic embolism. Cardiology Journal. 2009;16(6):560-3.

7. Basso C, Bottio T, Rubino M, Ruffatti A, Pittarello D, Thiene G, et al. Antiphospholipid syndrome and right atrial mass. J Thorac Cardiovasc Surg. 2005;130(5):1462-3.

8. Polytarchou K, Varvarousis D, Manolis AS. Cardiovascular Disease in Antiphospholipid Syndrome. Current Vascular Pharmacology. 2020.

9. Waisayarat J, Plumworasawat S, Vilaiyuk S, Sirachainan N. Intracardiac thrombus in a patient with catastrophic antiphospholipid syndrome: an autopsy case report and review of the literature. Vascular health and risk management. 2019;15:253.

10. Amaya-Amaya J, Rojas-Villarraga A, Anaya J-M. Cardiovascular disease in the antiphospholipid syndrome. Lupus. 2014;23(12):1288-91.

11. Zuily S, Huttin O, Mohamed S, Marie P-Y, Selton-Suty C, Wahl D. Valvular heart disease in antiphospholipid syndrome. Current rheumatology reports. 2013;15(4):320.

12. Cianciulli TF, Saccheri MC, Redruello HJ, Cosarinsky LA, Celano L, Trila CS, et al. Right atrial thrombus mimicking myxoma with pulmonary embolism in a patient with systemic lupus erythematosus and secondary antiphospholipid syndrome. Texas Heart Institute Journal. 2008;35(4):454.

13. Cianciulli TF, Saccheri MC, Lax JA, Neme RO, Sevillano JFA, Maiori ME, et al. Left ventricular thrombus mimicking primary cardiac tumor in a patient with primary antiphospholipid syndrome and recurrent systemic embolism. Cardiology Journal. 2009;16(6):560-3. 
14. Basso C, Bottio T, Rubino M, Ruffatti A, Pittarello D, Thiene G, et al. Antiphospholipid syndrome and right atrial mass. J Thorac Cardiovasc Surg. 2005;130(5):1462-3.

15. Naseer U, Hamza M, Shahzad SR, Nazeer H, Mookherjee S. CORONARY EMBOLI IN A PATIENT WITH INTRACARDIAC THROMBUS: AN UNUSUAL MANIFESTATION OF ANTI-PHOSPHOLIPID SYNDROME. Chest. 2020;158(4):A204.

16. Ye Z-X, Yu W-C, Hsueh C-M, Leu H-B, Chen J-W, Lin S-J. Antiphospholipid syndrome presenting as intracardiac thrombus with pulmonary embolism. Circulation Journal. 2005;69(10):1290-2.

17. Voigtlaender M, Conradi L, Hinsch A, Langer F. Right atrial thrombosis in antiphospholipid syndrome with secondary immune thrombocytopenia. The Thoracic and cardiovascular surgeon reports. 2015;4(1):40.

18. Zahid H, Hassan S, Gul S, Rizwan K, Khan SU, Maghazi MA. Co-existing bilateral pulmonary embolism and intra-cardiac mass: A case of catastrophic antiphospholipid syndrome-like disease. Cureus. 2018;10(10).

19. Permanyer E, Alegret J, Muñoz-Guijosa C, Padró J. Inferior vena cava obstruction by a cardiac mass: unusual presentation of primary antiphospholipid syndrome. The Thoracic and cardiovascular surgeon. 2011;59(03):182-3.

20. Dhibar DP, Sahu KK, Varma SC, Kumari S, Malhotra P, Mishra AK, et al. Intra-cardiac thrombus in antiphospholipid antibody syndrome: an unusual cause of fever of unknown origin with review of literature. Journal of Cardiology Cases. 2016;14(5):153-6. 

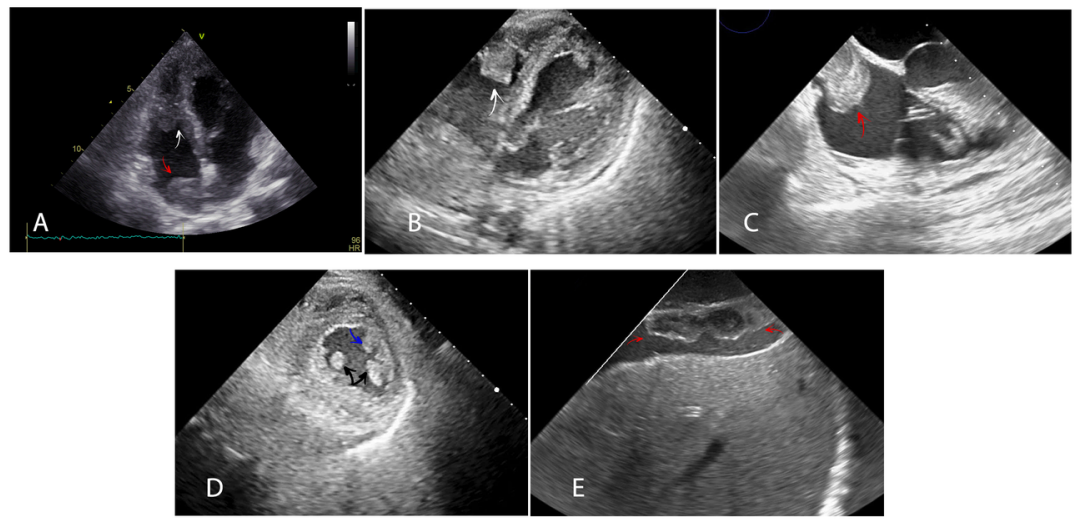

Figure 1: Transthoracic and transesophageal echocardiographic examinations of the patient

A) Transthoracic echocardiography in the 4-chamber view shows a large right atrial mass (red arrow) and an intracavity right ventricular mass (white arrow). (B) Transthoracic echocardiography in the subxiphoid view demonstrates a right ventricular mass (white arrow) with obliteration into right ventricular free wall. (C) Transesophageal echocardiography in the mid-esophageal view illustrates a large homogenous right atrial mass (red arrow) in the superior part of the right atrium. (D) Transthoracic echocardiography in the parasternal short-axis view depicts 2 papillary muscles (black arrow) and a small right ventricular mass (blue arrow). (E) Transthoracic echocardiography in the subxiphoid view presents dilation in the inferior vena cava with a large lobulated mass (red arrow). 

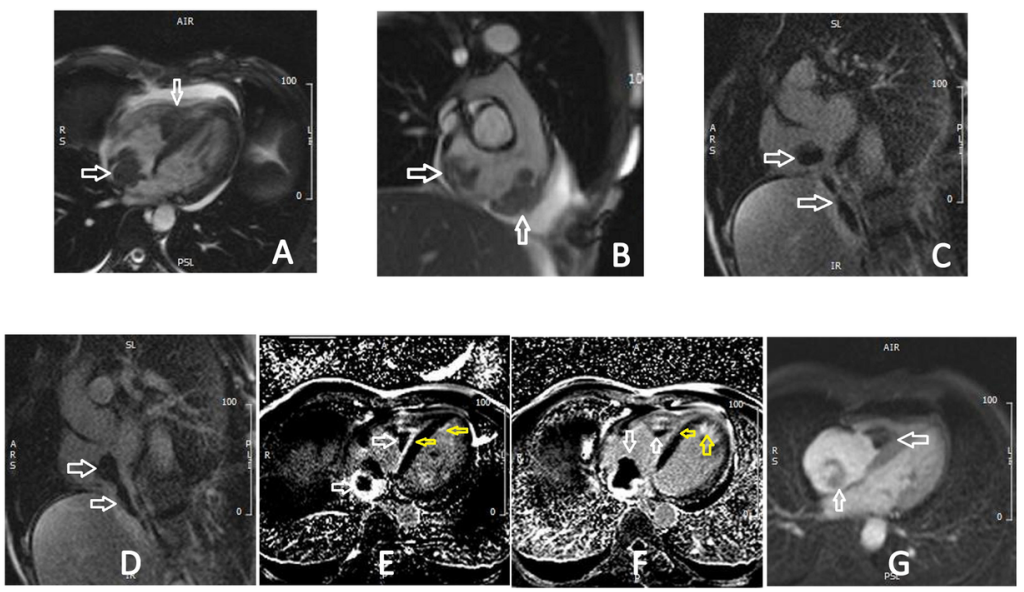

Figure 2: Comprehensive cardiac magnetic resonance imaging with a special tumor protocol

A) Steady-state free precession (SSFP) sequence in the 4-chamber view shows 2 hyposignal mass lesions (white arrow) in the right atrium and right ventricle. (B) Inflow-outflow right ventricular SSFP sequence illustrates 2 hyposignal mass lesions (white arrow) in the right atrium and right ventricle. (C \& D) Phase-sensitive inversion recovery (PSIR) sequence demonstrates long segment filling defects in the inferior vena cava and the right atrium. (E \& F) PSIR sequence depicts subendocardial late gadolinium enhancement (yellow arrow) in the right ventricle and left ventricular apex and 2 hyposignal mass lesions (white arrow) in the right atrium and right ventricle. (G) Early perfusion sequence illustrates 2 hyposignal mass lesions (white arrow) in the right atrium and right ventricle. 